\title{
LES DISPOSITIFS DE FRANCHISSEMENT : BASES BIOLOGIQUES, LIMITES ET RAPPELS RÉGLEMENTAIRES
}

\author{
J.P. PORCHER ${ }^{\star}$ et F. TRAVADE ${ }^{\star \star}$
}

\begin{abstract}
* Conseil Supérieur de la Pêche - DR 2 - 84, Rue de Rennes - 35510 CESSON SEVIGNE
** EDF - Etudes et Recherches - 6, Quai Watier - 78401 CHATOU CEDEX
\end{abstract}

\section{LES POISSONS MIGRATEURS : QUELQUES DÉFINITIONS}

Les populations de poissons dépendent très étroitement des caractéristiques de l'habitat aquatique : celui-ci est le support de toutes les fonctions biologiques (reproduction, nutrition, locomotion...).

Cette dépendance est exacerbée chez les poissons migrateurs qui exigent des milieux différents pour le déroulement des phases principales de leur cycle biologique : reproduction, production de juvéniles, grossissement et production de géniteurs. La possibilité de circuler d'un milieu à l'autre est obligatoire pour la survie de l'espèce.

On distingue deux grands groupes d'espèces migratrices:

- les migrateurs holobiotiques réalisent leur cycle biologique entièrement en eau douce : les zones de reproduction et les zones de grossissement sont plus ou moins éloignées.

Toutes les espèces se déplacent dans le réseau hydrographique, mais cette activité migratoire est plus ou moins importante pour le bon déroulement de leur cycle biologique. Chez des espèces telles que le brochet, la truite fario, la truite de lac, les zones indispensables aux phases successives du cycle biologique sont bien individualisées et souvent séparées par des distances importantes : les besoins migratoires sont stricts pour le maintien d'une population en bon état. Chez d'autres espèces telles que l'ablette ou le gardon, ces besoins sont moins marqués mais il est nécessaire de maintenir une circulation d'individus entre les biefs pour éviter l'isolement reproducteur.

- les migrateurs amphibiotiques doivent obligatoirement changer de milieu au cours de leur cycle qui se déroule pour partie en eau douce et pour partie en mer, avec des trajets entre zones de reproduction et zones de grossissement pouvant atteindre plusieurs milliers de kilomètres.

A chaque changement de milieu de vie, les migrateurs amphibiotiques subissent des transformations physiologiques et morphologiques qui les rendent fragiles. Chez les juvéniles de salmonidés migrateurs, cette transformation porte le nom de smoltification. Elle leur permet de s'adapter à la phase de vie marine.

Chez les migrateurs amphibiotiques, on distingue à nouveau deux groupes :

- les migrateurs potamotoques (saumon, alose, lamproie, esturgeon), dont la reproduction a lieu en eau douce et la phase de grossissement en milieu marin. La migration continentale est une migration de reproduction.

- les migrateurs thalassotoques (anguille), pour lesquels le schéma est inverse. La migration continentale est une migration trophique de colonisation.

Les migrateurs potamotoques savent reconnaître leur réseau hydrographique natal et viennent s'y reproduire avec un taux d'erreur très faible. Ce phénomène de retour au foyer ("homing") repose principalement surl'identification olfactive du cours d'eau. Il a pour conséquence 
que chaque bassin hydrographique possède un stock qui lui est propre et qui constitue une unité de gestion incontournable.

Dans le cas de l'anguille, les géniteurs se rassemblent dans la mer des Sargasses et on n'observe pas d'isolement reproducteur des individus issus de tel ou tel bassin : le stock d'anguilles est commun à l'ensemble de la façade atlantique européenne.

\section{LES OBSTACLES A LA CIRCULATION : PRINCIPAL FACTEUR HISTORIQUE DE LA RÉGRESSION DES ESPĖCES MIGRATRICES}

On a assisté en France au cours des derniers siècles à une réduction constante de l'aire de distribution des poissons migrateurs, réduction qui s'est accélérée avec le temps jusqu'à une période très récente.

La régression des espèces migratrices a été particulièrement importante chez les espèces amphibiotiques pour lesquelles le changement de milieu est obligatoire. Dans la grande majorité des cas, la cause essentielle de la régression ou de la disparition des migrateurs a été la construction d'obstacles à la circulation. Leur impact a jusqu'ici éclipsé très largement celui de la pollution des eaux ou des prélèvements par pêche. Ces obstacles sont la cause de la disparition de stocks entiers (saumon du Rhin, de la Seine, de la Garonne...), ou du cantonnement de certaines espèces dans une partie très réduite du réseau hydrographique (saumon de la Loire, alose de la Garonne ou du Rhône...).

Ce n'est que très récemment (décennie 1970), et grâce à la mise au point et à la mise en oeuvre d'une technologie spécifique, que la tendance s'est inversée et que l'on a pu envisager la reconquête de territoires pour les migrateurs. Ce savoir-faire a permis la mise en place de programmes de restauration ou de développement des stocks de migrateurs.

\section{LES EXIGENCES EN MATIÈRE DE LIBRE CIRCULATION}

\subsection{Les besoins de circulation varient suivant les espèces}

Le tableau I donne les caractéristiques biologiques des principales espèces migratrices amphibiotiques. Les zones de reproduction des espèces potamotoques sont réparties sur l'ensemble du réseau hydrographique : zones supérieures et moyennes pour les salmonidés, zones moyennes et inférieures pour les aloses et les lamproies. La colonisation du réseau hydrographique par l'anguille concerne l'ensemble du bassin.

Les bassins de la façade atlantique sont ainsi susceptibles d'accueillir les différents stades de l'une ou l'autre espèce migratrice dans la quasi-totalité de leur étendue.

La restauration ou le maintien d'un stock de migrateurs ne peut aboutir que si les zones adéquates sont accessibles. Les stratégies d'aménagement devront tenir compte de la situation de l'obstacle dans le bassin et des espèces présentes :

- pour les tronçons de cours d'eau situés à l'aval des zones de reproduction (cas du saumon ou de la truite de mer), il est nécessaire de maintenir une libre circulation permanente et totale dès lors que des migrateurs sont présents au pied des obstacles. Tous les ouvrages sans exception doivent être aménagés et les retards de migration au niveau de chaque obstacle doivent être d'autant plus réduits que l'axe migratoire est long et qu'il présente des aménagements nombreux,

- lorsque les obstacles sont situés au sein des zones de grossissement ou de reproduction, l'aménagement des obstacles les plus aval débouche sur un gain de production immédiat. L'anguille et l'alose illustrent ce cas de figure. II est également nécessaire pour ces espèces de minimiser les délais de franchissement des obstacles car leur période d'activité migratoire est limitée dans le temps et, pour l'alose, très proche de la date de reproduction,

- dans le cas des espèces holobiotiques à besoins migratoires limités (notamment les cyprinidés), il est nécessaire de maintenir une communication entre les biefs, mais le nombre de migrateurs à faire passer ne revêt pas la même importance. On attachera une attention particulière aux conditions hydrauliques dans l'ouvrage mais on peut tolérer une attractivité plus faible de la passe à poissons si elle n'est destinée qu'à ces espèces. 
Tableau I : Caractéristiques du cycle des principaux migrateurs amphibiotiques.

Table I : Characteristics of life cycle of most common migratory species.

\begin{tabular}{|c|c|c|c|c|c|c|c|c|c|c|c|}
\hline 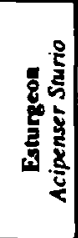 & 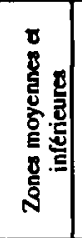 & 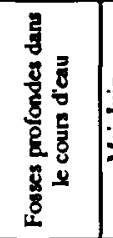 & 竞 & 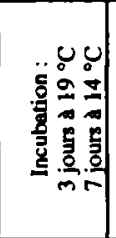 & 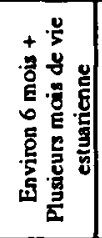 & 递 & 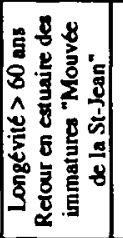 & 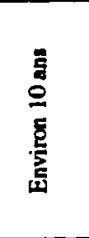 & $\mid \begin{array}{l}\frac{5}{3} \\
\frac{3}{2} \\
\frac{3}{2}\end{array}$ & छี & \\
\hline 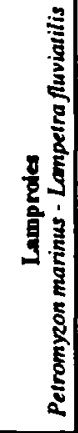 & 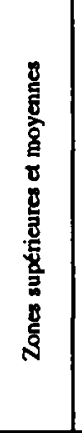 & 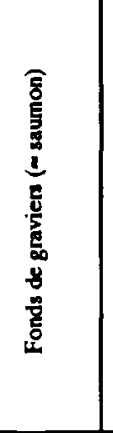 & 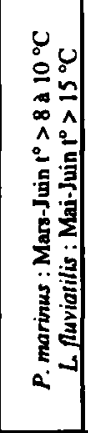 & 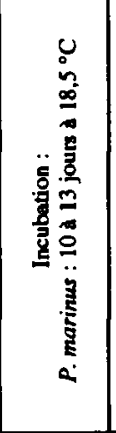 & 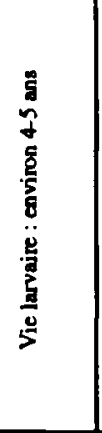 & 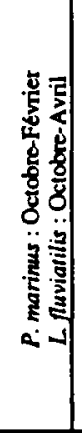 & 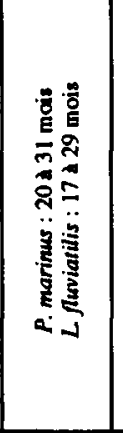 & $\stackrel{\overrightarrow{\mathbf{g}}}{\stackrel{\dot{b}}{\dot{b}}}$ & 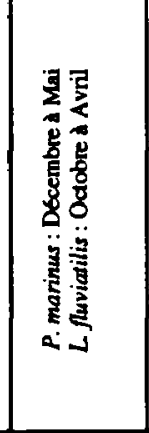 & $\frac{8}{2}$ & 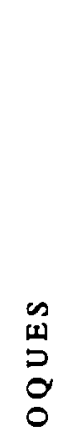 \\
\hline 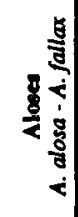 & 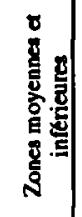 & 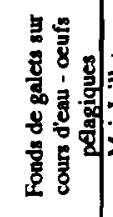 & $\frac{2}{2}$ & 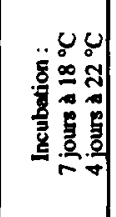 & 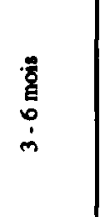 & 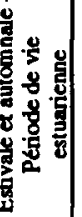 & 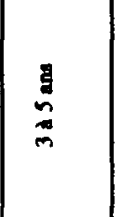 & 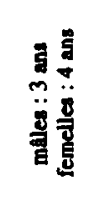 & 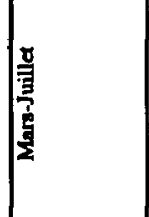 & 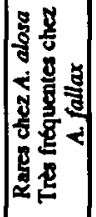 & $\begin{array}{l}0 \\
\vdots \\
\Sigma \\
5 \\
0 \\
0 \\
0\end{array}$ \\
\hline 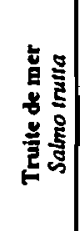 & 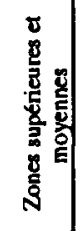 & 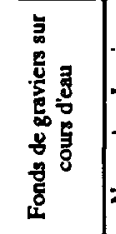 & 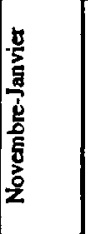 & 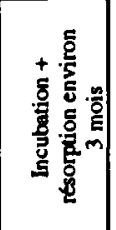 & 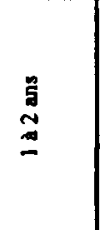 & 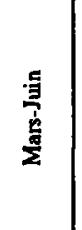 & 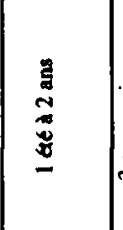 & 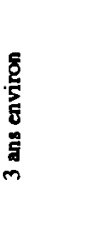 & 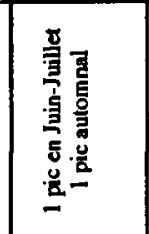 & 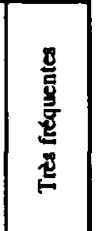 & 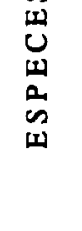 \\
\hline 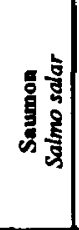 & 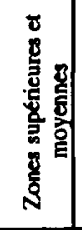 & 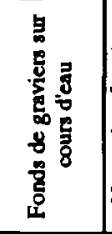 & 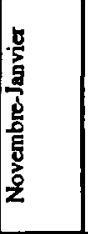 & 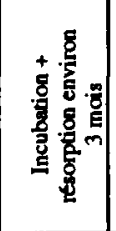 & $\begin{array}{l}\stackrel{\ddot{s}}{\Xi} \\
\stackrel{\Xi}{\Xi}\end{array}$ & $\frac{a}{\frac{3}{2}}$ & $\stackrel{\Xi}{\beth}$ & 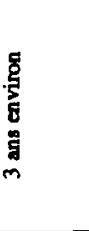 & 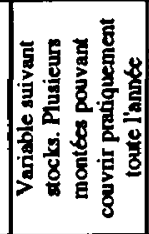 & $\begin{array}{l}\frac{9}{9} \\
\frac{0}{v} \\
\frac{8}{3} \\
3\end{array}$ & \\
\hline \multirow{2}{*}{ 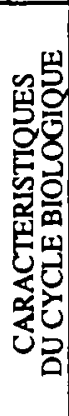 } & 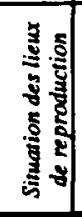 & 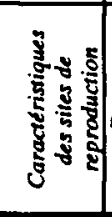 & zั & 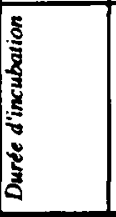 & 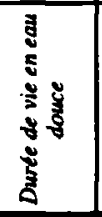 & $\frac{5}{\frac{8}{3}}$ & 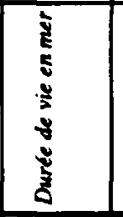 & 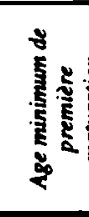 & 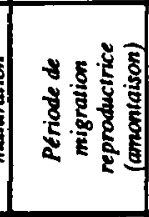 & 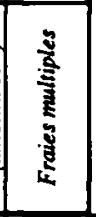 & \\
\hline & 3 & & 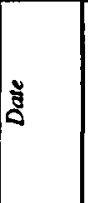 & 绻 & 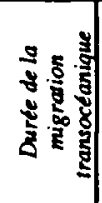 & 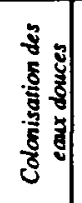 & 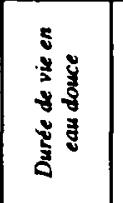 & 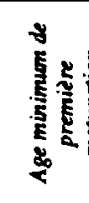 & 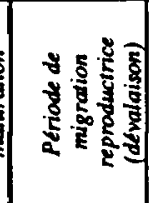 & 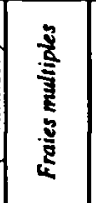 & \\
\hline 总 & & 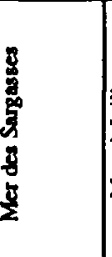 & 急 & - & $\begin{array}{l}\frac{5}{\ddot{8}} \\
\frac{8}{8} \\
\frac{6}{5}\end{array}$ & 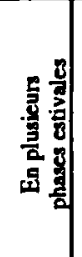 & 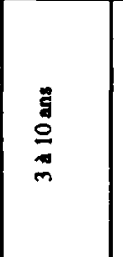 & 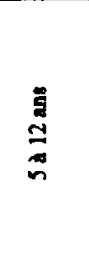 & 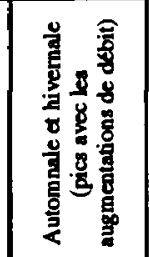 & ह̊ & 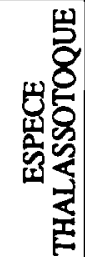 \\
\hline
\end{tabular}




\subsection{La libre circulation est une exigence quasi permanente}

Sur les grands cours d'eau, le chevauchement des périodes migratoires des multiples espèces présentes oblige à maintenir les obstacles franchissables en permanence. Ceci est illustré par la figure 1 qui représente les effectifs moyens mensuels de migrateurs dénombrés sur la Dordogne dans la passe à poissons de Bergerac. L'important pic printanier (avril-juin) correspond à la migration reproductrice de l'alose et au passage d'espèces holobiotiques. En dehors de cette pointe, la circulation des poissons est quasi continue, avec concentration de certaines espèces à des périodes préférentielles (par exemple saumons et truites de mer en fin d'année : octobre à décembre). II faut noter que la conception du dispositif de piégeage de Bergerac ne permet pas de contrôler le passage des lamproies et de l'anguille, qui augmenteraient les effectifs printaniers et estivaux.

Sur les cours d'eau à saumons, il existe des remontées successives qui s'étalent sur plusieurs saisons, et peuvent couvrir la totalité de l'année lorsque les conditions hydroclimatiques sont favorables à la migration. La figure 2 donne les effectifs moyens mensuels de saumons capturés à la station de Kerhamon (rivière Elorn, Finistère). Le pic bien marqué des mois de mai, juin et juillet correspond à l'arrivée des grilses (saumons de court séjour marin très caractéristiques des cours d'eau du Massif Armoricain), L'activité migratoire se maintient avec une certaine variablité tout au long de l'année.

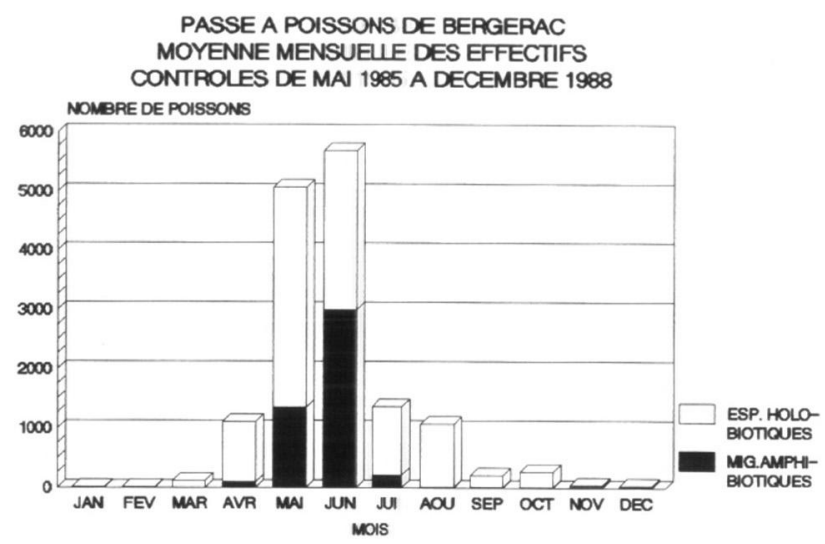

Figure 1 : Passages mensuels enregistrés à la passe à poissons de Bergerac sur la Dordogne.

Figure 1 : Monthly fish passage in Bergerac fishway on the Dordogne river.

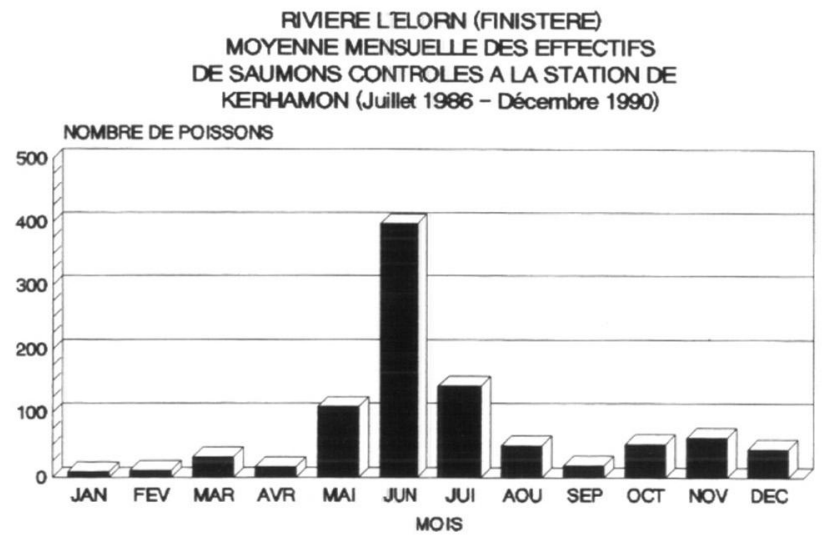

Figure 2 : Passages mensuels enregistrés à la station de contrôle de Kerhamon sur I'Elorn.

Figure 2 : Monthly fish passage at Kerhamon control station on the Elorn river. 
Compte tenu du nombre d'espèces présentes, ce n'est qu'exceptionnellement et pour des périodes peu durables qu'on peut se dispenser d'assurer le franchissement d'un obstacle. Ceci peut arriver dans les zones supérieures de certains bassins atteints seulement à l'approche de la période de reproduction des salmonidés par exemple. Mais, en tout état de cause, dès que des migrateurs sont présents au niveau d'un obstacle, la libre circulation doit être assurée. Les tentatives de franchissement d'un obstacle non équipé (ou mal équipé) provoquent des blessures ou des mortalités des migrateurs. Des observations réalisées sur des civelles et anguillettes bloquées à l'aval d'un barrage ont pu également mettre en évidence une mortalité importante par prédation.

\subsection{La libre circulation n'est jamais définitivement acquise}

La réalisation d'un dispositif de franchissement ne règle pas à elle seule le problème de la circulation des migrateurs. Ce dispositif doit être entretenu de façon permanente, et son efficacité doit être périodiquement vérifiée : tout changement de l'environnement est susceptible de remettre en cause son bon fonctionnement

- modification des lignes d'eau consécutives à des travaux ou à l'instabilité du lit,

- modifications dans la gestion hydraulique du site (modification des ouvrages, installation ou suppression de turbines, nouveaux modes de fonctionnement).

Le défaut d'un seul dispositif de franchissement sur l'axe migratoire suffit à ruiner la totalité des efforts consentis pour maintenir ou développer les stocks.

On devra donc considérer qu'un cours d'eau aménagé pour y rétablir la libre circulation doit être un cours d'eau sous surveillance permanente. II n'est plus assimilable à un système naturel et doit impérativement être géré.

\section{LE RÉTABLISSEMENT DE LA LIBRE CIRCULATION : MODE D'EMPLOI, INTÉRÊT ET LIMITES}

\subsection{L'aménagement des obstacles exige des compétences en biologie et en hydraulique}

Les exigences biologiques des espèces migratrices sont à la base de la conception des ouvrages de franchissement. Le terme de "passe à poissons" masque d'ailleurs la diversité des situations, et il faudrait en fait parler de passes à salmonidés, à aloses, à cyprinidés ou à anguilles. La connaissance des capacités physiques de nage ou de saut des migrateurs (fortement dépendantes de l'espèce, de la taille et de l'état physiologique des individus, de la température...), et celle de leur comportement face aux obstacles ont permis de définir des critères de conception et de dimensionnement des dispositifs de franchissement pour certaines espèces. Ces critères ne doivent désormais plus être ignorés par le projeteur. II devra en outre intégrer les informations propres au site à aménager et utiliser les compétences nécessaires en hydraulique et génie civil pour garantir le fonctionnement de l'ouvrage de franchissement dans toute la gamme de débits du cours d'eau habituelle en période de migration.

\subsection{L'équipement d'un obstacle avec une passe à poissons ne compense jamais en totalité les dommages causés aux migrateurs}

Le fait qu'un savoir-faire soit disponible en matière de rétablissement de la circulation des migrateurs ne doit pas laisser penser qu'on peut sans problème multiplier les barrages et les aménagements. D'une part, certaines nuisances apportées par la multiplication des équipements sur les cours d'eau sont irréparables (ennoiement de zones de frayères ou de production, modification de la qualité physico-chimique de l'eau, du régime hydrologique, ...). D'autre part, la passe à poissons la mieux conçue et la plus attractive induira toujours un retard de migration et l'effet cumulatif des aménagements sur un cours d'eau peut très vite atteindre des proportions non compatibles avec le maintien d'une population de migrateurs. Cet aspect est fondamental dans le cas des espèces exigeantes (aloses) et dans le cas de la migration d'avalaison qui peut s'accompagner de mortalités conséquentes au passage des turbines et évacuateurs de crue. 


\subsection{La libre circulation n'est qu'un élément de la gestion des stocks de migrateurs}

La libre circulation est une condition nécessaire au maintien ou à la restauration des populations de migrateurs. Si ce facteur a pu, dans le passé, être la cause primordiale de la régression ou de la disparition de certaines espèces, il n'est désormais qu'un élément parmi d'autres à prendre en compte dans la politique de gestion d'un stock.

Une population de migrateurs est régulée par de nombreux autres facteurs dont certains ont connu une évolution récente d'eau,

- dégradation de la qualité des eaux et modification des débits naturels des cours

—introduction ou dissémination de maladies ou parasites atteignant les espèces migratrices,

- altération de la qualité des habitats par les interventions physiques sur le cours d'eau (extractions de granulats, remodelages du lit), sur ses annexes (drainage de zones humides, isolement de plaines d'inondation), ou sur le bassin versant,

- développement de nouvelles pêcheries (sur les zones d'engraissement du saumon) ou accroissement de l'effort de pêche.

Une politique de gestion d'un stock de migrateurs ne peut être élaborée qu'après une évaluation soigneuse de tous ces facteurs. Elle doit traiter de front tous les problèmes, sous peine de voir l'un d'entre eux devenir limitant et ruiner tous les efforts consentis par ailleurs.

\subsection{Les dispositifs de franchissement doivent être évalués en fonction de leur intérêt biologique et économique et de leur coût}

Les objectifs des aménagements d'obstacles à la circulation sont :

- la minimisation des impacts de l'équipement hydraulique sur le cycle biologique des migrateurs dans le but de maintenir ou d'augmenter le stock,

— dans un nombre plus réduit de cas, la possibilité d'exploiter l'espèce sur un territoire plus vaste, dans la mesure où cette exploitation reste compatible avec le maintien du stock.

Dans chaque cas, on peut chercher à quantifier les bénéfices attendus et à les mettre en relation avec le coût du dispositif de franchissement. Une telle démarche permet :

(a) d'évaluer la "rentabilité biologique" de l'opération, qui dépend notamment de la position de l'équipement hydraulique dans l'aire de répartition de l'espèce. Si l'équipement d'un site qui se trouve à l'aval de toutes les zones de reproduction est strictement indispensable, on doit en revanche s'interroger sur la nécessité d'assurer le passage des géniteurs à l'amont d'un obstacle lorsque les zones de production situées à l'aval ne sont pas saturées et que la dévalaison des juvéniles n'est pas acquise sans mortalités substantielles,

(b) de définir un ordre de priorité dans la chronologie des chantiers lorsque de multiples ouvrages doivent être équipés sur des bassins ou des sous-bassins différents,

(c) de choisir entre plusieurs options d'équipement sur un même site, chacune d'entre elles présentant un "bénéfice biologique" et un coût propre.

Les principaux critères à prendre en compte sont les suivants :

— potentiels de reproduction et de production à l'amont de l'obstacle,

- le cas échéant, existence et degré de saturation des zones de production situées à l'aval de l'obstacle,

— besoins de dévalaison, et capacité d'assurer le passage de l'amont à l'aval au niveau de l'obstacle (mortalités prévisibles),

- coût des dispositifs de franchissement pour la montée et la dévalaison,

- existence et coûts de solutions alternatives de compensation (élevage et repeuplement, abandon d'aménagements obsolètes permettant de récupérer des zones de production,...).

La démarche à mettre en oeuvre pour des migrateurs potamotoques (reproduction dans le milieu eau douce) est résumée dans le diagramme porté sur la figure 3 . 
Pour certaines espèces (saumon, et dans une moindre mesure truite de mer), des outils de mesure des différents critères existent et permettent de mener la réflexion à son terme :

- estimation des capacités de production basée sur la description quantitative des habitats piscicoles,

- évaluation de la situation actuelle par le dénombrement des migrateurs, le recensement des frayères ou la réalisation d'inventaires piscicoles,

- estimation des possibilités de dévalaison et des mortalités probables au passage dans les turbines ou les évacuateurs de crue,

- estimation du coût de réalisation des différents équipements pour le rétablissement de la circulation (montée et dévalaison), ou des solutions alternatives de compensation.

En revanche, pour d'autres espèces (aloses, lamproies par exemple), l'état des connaissances est beaucoup moins avancé, et les éléments d'appréciation pourront manquer pour alimenter avec précision l'ensemble de la démarche.

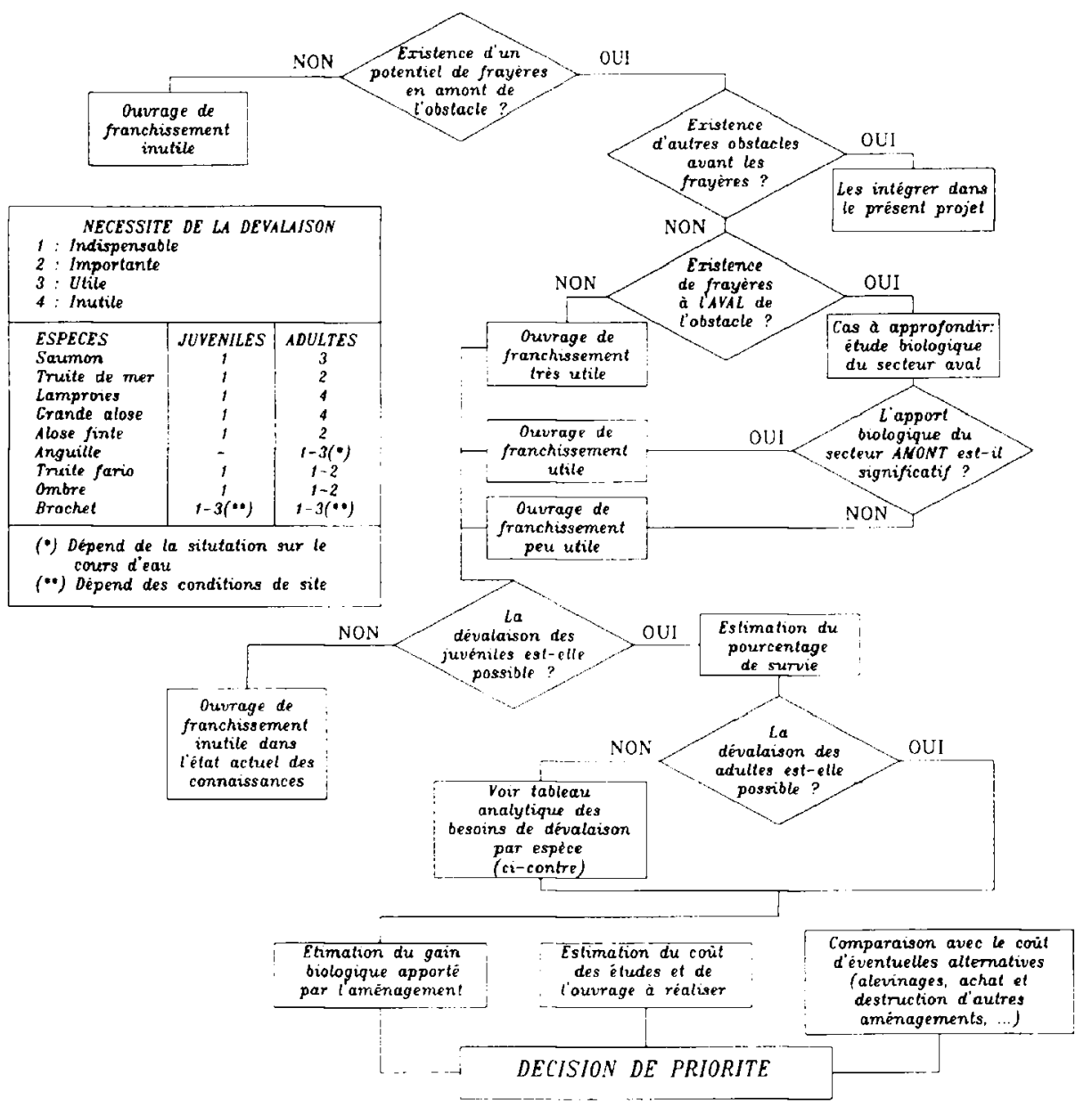

Figure 3 : Diagramme d'évaluation d'un aménagement pour restaurer la circulation des migrateurs holobiotiques ou amphibiotiques anadromes.

Figure 3 : Diagram for the evaluation of the need of a fish facility to restore fish passage for holobiotic and amphibiotic species. 


\title{
5. RAPPELS RÉGLEMENTAIRES
}

\subsection{Les principes}

Les articles L.232-5 à L.232-8 du Code Rural (cf. ci-après) définissent les obligations des permissionnaires à l'égard de la circulation des migrateurs et répriment les infractions.

Les exploitants d'ouvrages sont tenus à une obligation de résultat et doivent assurer en permanence :

- la vie, la circulation et la reproduction des poissons dans le tronçon court-circuité. Les espèces concernées sont celles qui peuplent les eaux au moment de l'installation de l'ouvrage.

- le franchissement des ouvrages dans les cours d'eau ou parties de cours d'eau dont la liste est fixée par décret, et pour les espèces définies par arrêté.

Un délai de mise en conformité de 5 années après le classement des cours d'eau et la parution de la liste des espèces est prévu pour les ouvrages existants.

La loi définit un débit minimal à maintenir dans le lit des cours d'eau, débit lui-même assujetti à l'obligation de résultat. La mise à disposition de ce débit, ou la réalisation des aménagements rendus nécessaires pour satisfaire aux dispositions de la loi n'ouvrent pas droit à indemnité.

Enfin, les circulaires d'application de ces textes précisent :

- d'une part que la réglementation d'ouvrages sans existence juridique, ainsi que le renouvellement d'autorisations ou de concessions sont assimilés à des créations et doivent remplir les conditions définies par la loi,

- d'autre part, que l'administration doit imposer aux nouveaux ouvrages les dispositifs de franchissement qui s'avèrent nécessaires aux espèces migratrices présentes ou en cours de réintroduction, y compris sur les cours d'eau non classés au titre de l'article L.232-6 du Code Rural.

\subsection{Les textes}

\section{CODE RURAL \\ TITRE III : PÊCHE EN EAU DOUCE ET GESTION DES RESSOURCES PISCICOLES}

\author{
Chapitre II : Préservation des milieux aquatiques \\ et protection du patrimoine piscicole \\ Section 3 \\ Obligations relatives aux ouvrages
}

\section{Article L.232-5}

Tout ouvrage à construire dans le lit d'un cours d'eau doit comporter des dispositifs maintenant dans ce lit un débit minimal garantissant en permanence la vie, la circulation et la reproduction des espèces qui peuplent les eaux au moment de l'installation de l'ouvrage ainsi que, le cas échéant, des dispositifs empêchant la pénétration du poisson dans les canaux d'amenée et de fuite.

Ce débit minimal ne doit pas être inférieur au dixième du module du cours d'eau au droit de l'ouvrage correspondant au débit moyen interannuel, évalué à partir des informations disponibles portant sur une période minimale de cinq années, ou au débit à l'amont immédiat de l'ouvrage, si celui-ci est inférieur.

Toutefois, pour les cours d'eau ou parties de cours d'eau dont le module est supérieur à 80 mètres cubes par seconde, des décrets en Conseil d'Etat pourront, pour chacun d'eux, fixer à ce débit minimal une limite inférieure qui ne devra pas se situer en dessous du vingtième du module.

L'exploitant de l'ouvrage est tenu d'assurer le fonctionnement et l'entretien des dispositifs garantissant dans le lit du cours d'eau le débit minimal défini aux deux alinéas précédents. 
Les dispositions prévues aux alinéas précédents seront étendues aux ouvrages existant au 30 juin 1984 par réduction progressive de l'écart par rapport à la situation actuelle. Ces dispositions s'appliqueront intégralement au renouvellement des concessions ou autorisations de ces ouvrages.

Dans un délai de trois ans à compter du 30 juin 1984, leur débit minimal devra, sauf impossibilité technique inhérente à leur conception, être augmenté de manière à atteindre le quart des valeurs fixées aux deuxième et troisième alinéas du présent article. Dans un délai de cinq ans, le Gouvernement présentera au Parlement un bilan de l'application du présent alinéa.

L'application des dispositions du présent article ne donne lieu à aucune indemnité.

Les dispositions du présent article ne s'appliquent pas au Rhin et au Rhône en raison du statut international de ces deux fleuves.

Article L.232-6

Dans les cours d'eau ou parties de cours d'eau et canaux dont la liste est fixée par décret, après avis des conseils généraux rendus dans un délai de six mois, tout ouvrage doit comporter des dispositifs assurant la circulation des poissons migrateurs. L'exploitant de l'ouvrage est tenu d'assurer le fonctionnement et l'entretien de ces dispositifs.

Les ouvrages existants doivent être mis en conformité, sans indemnité, avec les dispositions du présent article dans un délai de cinq ans à compter de la publication d'une liste d'espèces migratrices par bassin ou sous-bassin fixée par le ministre chargé de la pêche en eau douce et, le cas échéant, par le ministre chargé de la mer.

\section{Article L.232-7}

Le classement des cours d'eau, parties de cours d'eau et canaux intervenu au titre du régime des échelles à poissons antérieurement au 1er janvier 1986 vaut classement au titre du premier alinéa de l'article L.232-6.

\section{Article L. 232-8}

Ceux qui ne respectent pas les dispositions des articles L.232-5 et L.232-6 seront punis d'une amende de $1000 \mathrm{~F}$ à $80000 \mathrm{~F}$. Lorsqu'une personne est condamnée en application du présent article, le tribunal peut décider que le défaut d'exécution, dans le délai qu'il fixe, des mesures qu'il prescrit aux fins prévues aux articles susmentionnés entraînera le paiement d'une astreinte définie à l'article L.238-7.

\section{NOTION D'EFFICACITÉ DES PASSES A POISSONS}

L'obligation de résultats imposée par la loi en matière de libre circulation débouche sur la notion d'efficacité des ouvrages de franchissement, qui est actuellement mal définie. Une passe efficace pour une espèce donnée est-elle celle où il a transité au moins un individu ? ou toute la population présente au pied de l'obstacle ? ou bien une fraction définie de cette population ? la durée que met une population à franchir l'obstacle (retard à la migration) doit elle prise en compte?

La formulation n'en est pas simple car la notion d'efficacité doit être mise en rapport avec la nécessité (ou l'intérêt) biologique de l'ouvrage. Elle est donc relative aux espèces considérées, au nombre d'obstacles sur les cours d'eau et à la position de l'obstacle sur ce cours d'eau.

Dans une passe à saumons ou truites de mer située à l'aval des zones de frayères, on cherchera à faire transiter la totalité de la population migrante. Si de surcroît ce cours d'eau est équipé d'un grand nombre d'obstacles, on cherchera en plus à minimiser le temps mis par les poissons pour l'emprunter de façon à ce qu'ils arrivent à temps sur les frayères. Si, par contre, cette passe est située à l'amont du cours d'eau au milieu de la zone de frayères on pourra être moins exigeant sur son efficacité.

Pour une passe à aloses, espèce caractérisée par une durée de migration très courte, l'efficacité de la passe résidera notamment dans la minimisation du retard au droit de l'obstacle. 
Enfin, pour une passe à cyprinidés dont l'intérêt biologique est avant tout d'éviter la sectorisation des populations dans les différents biefs, il n'est pas nécessaire de chercher à faire transiter la totalité des populations présentes à l'aval d'un obstacle. La passe sera efficace si un "certain nombre" d'individus (en proportion "raisonnable" par rapport à la taille des populations) y transite.

Le niveau d'efficacité à atteindre pour les passes à poissons dépend aussi du degré d'aménagement du bassin : sur un cours d'eau comptant 3 obstacles à la migration, l'installation de passes à poissons ayant une efficacité de $60 \%$ assure le passage de 1/5 des migrateurs qui se présentent à l'embouchure. Sur un cours d'eau comptant 30 obstacles, il faudrait que tous les dispositifs de franchissement aient une efficacité de $95 \%$ pour arriver au même résultat.

Il en va de même vis-à-vis de l'efficacité de dispositifs de dévalaison destinés à minimiser les mortalités des juvéniles lors de leur transit dans les turbines ou les déversoirs de crue. Sur un cours d'eau où un certain pourcentage de mortalité par obstacle est jugé comme biologiquement acceptable, un ouvrage de dévalaison devra être d'autant plus efficace que la mortalité potentielle dans les turbines est élevée. Si on accepte par exemple $10 \%$ de mortalité moyenne par usine sur un cours d'eau et que la mortalité de transit dans l'usine A est de $20 \%$, l'efficacité exigible pour l'ouvrage de dévalaison est de $50 \%$. Pour l'usine B dont la mortalité de transit serait de $60 \%$, l'efficacité exigible pour l'ouvrage de dévalaison est alors de $83 \%$.

Par ailleurs, en fonction d'un objectif de mortalité maximale pour l'ensemble d'un bassin, l'exigence en matière d'efficacité des ouvrages de dévalaison est d'autant plus forte que le nombre d'obstacles est élevé.

D'une manière générale, l'impact potentiel d'un aménagement sur une population de migrateurs est d'autant plus marqué qu'il est situé plus bas sur le cours d'eau. Une attention toute particulière doit donc être portée à l'efficacité, tant pour la montée que pour la dévalaison, des aménagements situés dans la partie inférieure des cours d'eau.

En conclusion, le niveau d'efficacité à atteindre sur un site donné doit être défini par rapport aux objectifs biologiques recherchés, et non dans l'absolu. II sera donc fixé en tenant compte de la situation de l'ouvrage dans le bassin, des capacités à assurer la dévalaison sur le site, et de l'impact des autres obstacles. Les objectifs définis pour l'ensemble du bassin ne pourront alors être atteints que par un accord dans l'action entre les exploitants d'ouvrage et les gestionnaires des populations piscicoles. 